\title{
The Order of the $p$-Selmer Groups and the Rank of Elliptic Curves
}

\author{
Hisayoshi SATO \\ Kyushu University \\ (Communicated by K. Katayama)
}

\section{Introduction.}

Let $E$ be an elliptic curve defined over a number field $k$. Then the set $E(k)$ of all $k$-rational points of $E$ is a finitely generated abelian group. By the rank of $E / k$ we mean the rank of the free part of $E(k)$. The rank is deeply connected with the order of the Selmer group. In this paper, we give an upper bound of the order of the $p$-Selmer group of $E / k$ for a prime number $p$ in terms of the ideal class group of certain finite extension of $k$. There are various results about the order of the Selmer groups. Brumer-Kramer [2], Washington [11], and Kawachi-Nakano [3] studied the case for $p=2$. For a cyclic isogeny $\phi$ of prime degree $p$, Aoki [1] estimated the order of the $\phi$-Selmer group by using the genus formula.

We here follow Aoki's method in order to estimate the order of the Selmer group for the multiplication-by- $p$ map.

In Section 2, we recall the general facts about the Selmer group and define some maps which will be needed later. In Section 3, we embed the $p$-Selmer group for an odd prime $p$ in some Galois cohomology groups and estimate the order of the Selmer group by making use of the genus formula under some assumptions. For $p=2$, we discuss in Section 4. In Section 5, we show that the assumptions in Section 3 hold for an elliptic curve without complex multiplication whenever we choose a suitable prime number $p$ and replace the base field $k$ by some finite extension of $k$.

\section{Preliminaries.}

Let $k$ be an algebraic number field of finite degree and $E$ be an elliptic curve defined over $k$. For any integer $m \geq 2, E[m]$ denotes the kernel of the multiplication-by- $m$ map $[m]$. Let $S$ be a finite set of places of $k$ consisting of the infinite places, those which divide $m$ and those at which $E$ has bad reductions, and $k_{s}$ be the maximal Galois 
extension of $k$ which is unramified outside $S$. Then it is known that there is an exact sequence

$$
0 \longrightarrow H^{1}\left(k_{S} / k, E[m]\right) \longrightarrow H^{1}(k, E[m]) \longrightarrow \underset{v \in M_{k} \mid S}{\bigoplus^{1}} H^{1}\left(k_{v}, E\right)
$$

where $M_{k}$ means the set of all (finite, infinite) places of $k$ and $k_{v}$ denotes the completion of $k$ at $v \in M_{k}$ [5]. From the above sequence, the Selmer group $S^{(m)}(E / k)$ of $E / k$ for [m] is given by

$$
\begin{aligned}
S^{(m)}(E / k) & =\operatorname{Ker}\left\{H^{1}(k, E[m]) \rightarrow \prod_{v \in M_{k}} H^{1}\left(k_{v}, E\right)\right\} \\
& =\operatorname{Ker}\left\{H^{1}(k, E[m]) \rightarrow \prod_{v \in S} H^{1}\left(k_{v}, E\right)\right\} \cap \operatorname{Ker}\left\{H^{1}(k, E[m]) \rightarrow \prod_{v \notin S} H^{1}\left(k_{v}, E\right)\right\} \\
& =\operatorname{Ker}\left\{H^{1}\left(k_{S} / k, E[m]\right) \rightarrow \prod_{v \in S} H^{1}\left(k_{v}, E\right)\right\} .
\end{aligned}
$$

The following diagram is commutative:

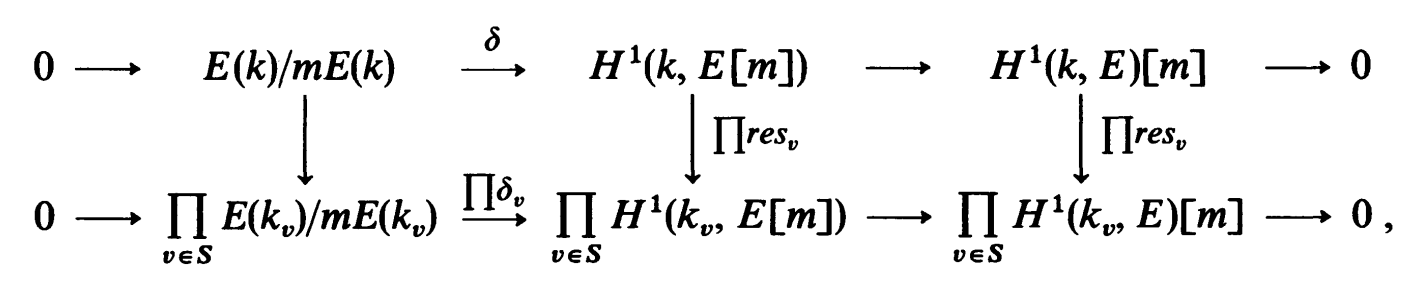

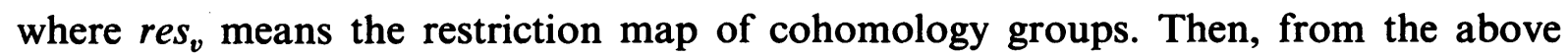
discussion, the Selmer group is given by

$$
S^{(m)}(E / k)=\left\{\xi \in H^{1}\left(k_{S} / k, E[m]\right) \mid \operatorname{res}_{v}(\xi) \in \operatorname{Im} \delta_{v} \text { for any } v \in S\right\}
$$

Next suppose that $E[m]=\langle P\rangle \times\left\langle P^{\prime}\right\rangle \subset E(k)$, where $P, P^{\prime}$ are some generators over $\mathbf{Z} / m \mathbf{Z}$. Let $\mu_{m} \subset k^{\times}$be the group of $m$-th roots of unity and $e_{m}$ be the Weil pairing

$$
e_{m}: E[m] \times E[m] \longrightarrow \mu_{m}
$$

For fixed generators $P, P^{\prime}$ of $E[m]$ over $Z / m Z$, it is easily seen that $e_{m}$ gives an isomorphism

$$
\begin{gathered}
i: H^{1}(k, E[m]) \stackrel{\sim}{\longrightarrow} H^{1}\left(k, \mu_{m}\right) \times H^{1}\left(k, \mu_{m}\right), \\
\xi \longmapsto\left(\left[\sigma \mapsto e_{m}\left(\xi(\sigma), P^{\prime}\right)\right],\left[\tau \mapsto e_{m}(P, \xi(\tau))\right]\right) .
\end{gathered}
$$

Let $\kappa$ be the isomorphism given by the Kummer sequence for the field $k$ :

$$
\kappa: H^{1}\left(k, \mu_{m}\right) \stackrel{\sim}{\longrightarrow} k^{\times} / k^{\times m} .
$$

Then using the isomorphisms $\kappa$ and $i$, we define an isomorphism 


$$
\begin{gathered}
j: H^{1}(k, E[m]) \stackrel{\sim}{\longrightarrow}\left(k^{\times} / k^{\times m}\right)^{2}, \\
j=(\kappa \times \kappa) \circ i .
\end{gathered}
$$

For any place $v$ of $k$, let $i_{v}, \kappa_{v}, j_{v}$ denote the maps which are defined over $k_{v}$ in a similar way as $i, \kappa, j$ respectively. Moreover, $e_{m}$ defines a cup product

$$
\langle,\rangle_{v}: H^{1}\left(k_{v}, E[m]\right) \times H^{1}\left(k_{v}, E[m]\right) \stackrel{\cup_{e_{m}}}{\longrightarrow} H^{2}\left(k_{v}, \mu_{m}\right) \stackrel{i n v_{v}}{\sim} \frac{1}{m} \mathbf{Z} / \mathbf{Z},
$$

where $i n v_{v}$ denotes the invariant map.

Finally, let $(,)_{v}: k_{v}^{\times} / k_{v}^{\times m} \times k_{v}^{\times} / k_{v}^{\times m} \rightarrow \mu_{m}$ be the Hilbert norm residue symbol. Then we define a bilinear map $\Phi$ as follows:

$$
\begin{gathered}
\Phi:\left(k_{v}^{\times} / k_{v}^{\times m}\right)^{2} \times\left(k_{v}^{\times} / k_{v}^{\times m}\right)^{2} \rightarrow \mu_{m}, \\
((a, b),(c, d)) \longmapsto(a, d)_{v}(b, c)_{v}^{-1} .
\end{gathered}
$$

LEMMA 1. The following diagram is commutative:

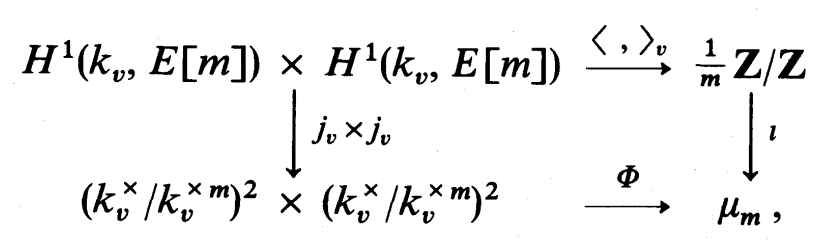

where $l(n / m)=e_{m}\left(P, P^{\prime}\right)^{n}$.

Proof. If we define a pairing

$$
\begin{gathered}
f: H^{1}\left(k_{v}, \mu_{m}\right)^{2} \times H^{1}\left(k_{v}, \mu_{m}\right)^{2} \longrightarrow H^{2}\left(k_{v}, \mu_{m}\right), \\
((\xi, \eta),(\phi, \psi)) \longmapsto\left[(\sigma, \tau) \mapsto(\xi \cup \psi)(\sigma, \tau)((\eta \cup \phi)(\sigma, \tau))^{-1}\right],
\end{gathered}
$$

then by the definition, it is easily checked that the following diagram is commutative:

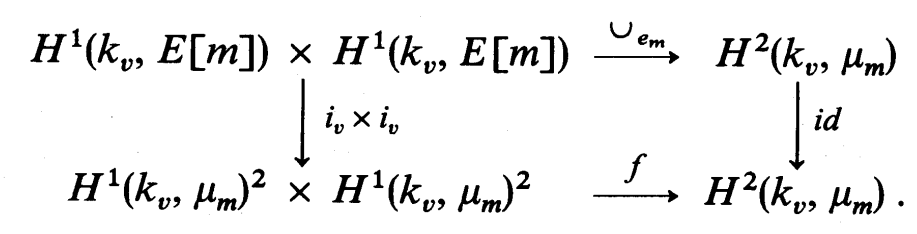

On the other hand, by [7] $\mathrm{Ch}$. XIV Proposition 5, we have a commutative diagram

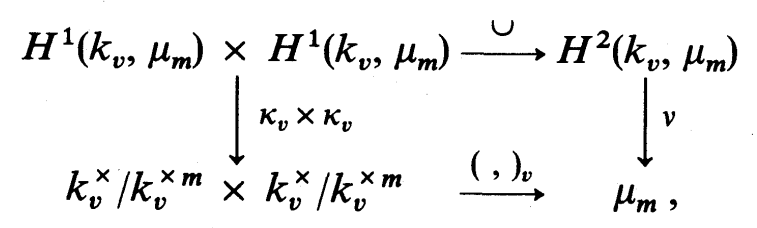


where $v$ stands for the composition of $i n v_{v}$ and $\imath: \frac{1}{m} \mathbf{Z} / \mathbf{Z} \stackrel{\sim}{\rightarrow} \mu_{m}$. Hence for $((\xi, \eta)$, $(\phi, \psi)) \in H^{1}\left(k_{v}, \mu_{m}\right)^{2} \times H^{1}\left(k_{v}, \mu_{m}\right)^{2}$, we have

$$
\begin{gathered}
v \circ f((\xi, \eta),(\phi, \psi))=v\left((\xi \cup \psi)(\eta \cup \phi)^{-1}\right)=v(\xi \cup \psi) v(\eta \cup \phi)^{-1} \\
=\left(\kappa_{v} \xi, \kappa_{v} \psi\right)_{v}\left(\kappa_{v} \eta, \kappa_{v} \phi\right)_{v}^{-1}=\Phi\left(\left(\kappa_{v} \xi, \kappa_{v} \eta\right),\left(\kappa_{v} \phi, \kappa_{v} \psi\right)\right)=\Phi \circ \kappa_{v}^{4}((\xi, \eta),(\phi, \psi)) .
\end{gathered}
$$

Therefore the diagram

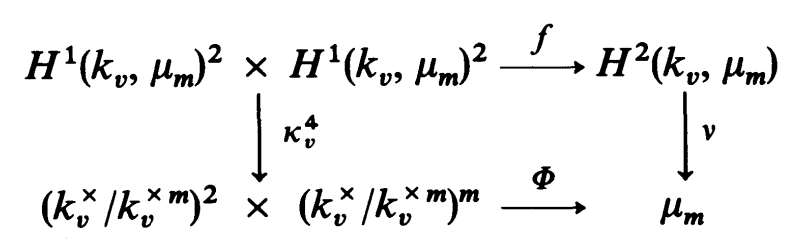

is commutative.

Moreover, the composite map $\frac{1}{m} \mathbf{Z} / \mathbf{Z} \stackrel{\text { inv }}{\longrightarrow} H^{2}\left(k_{v}, \mu_{m}\right) \stackrel{v}{\longrightarrow} \mu_{m}$ coincides with $\imath$ by the definition of $v$. This gives the desired result.

Taking account of Lemma 1 , let $\operatorname{Im} \delta_{v}^{\perp}$ be the annihilator of $\operatorname{Im} \delta_{v}$ with respect to $\langle,\rangle_{v}$, namely

$$
\operatorname{Im} \delta_{v}^{\perp}=\left\{\xi \in H^{1}\left(k_{v}, E[m]\right) \mid\langle\eta, \xi\rangle_{v}=0 \text { for any } v \in \operatorname{Im} \delta_{v}\right\}
$$

Then by the definition of $e_{m}$, it can be shown that $\operatorname{Im} \delta_{v} \subset \operatorname{Im} \delta_{v}^{\perp}$ [4].

On the other hand, the Tate pairing $E\left(k_{v}\right) \times H^{1}\left(k_{v}, E\right) \rightarrow \mathbf{Q} / \mathbf{Z}[10]$, [12] induces a perfect pairing

$$
E\left(k_{v}\right) / m E\left(k_{v}\right) \times H^{1}\left(k_{v}, E\right)[m] \rightarrow \frac{1}{m} \mathbf{Z} / \mathbf{Z},
$$

and this pairing is commutative with $\langle,\rangle_{v}$, namely the diagram

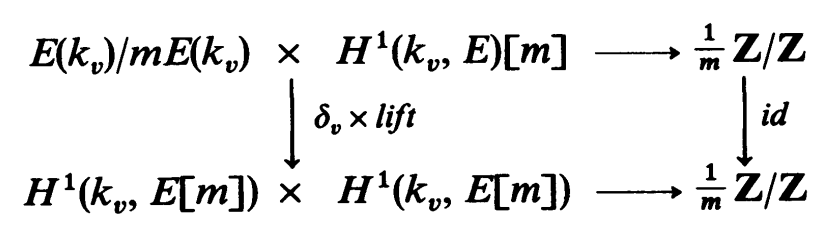

is commutative.

Hence, for any $\xi \in \operatorname{Im} \delta_{v}^{\perp} \subset H^{1}\left(k_{v}, E[m]\right)$, the image $\bar{\xi}$ in $H^{1}\left(k_{v}, E\right)[m]$ is an annihilator for $E\left(k_{v}\right) / m E\left(k_{v}\right)$. But the pairing is perfect, hence we have $\bar{\xi}=\overline{0}$. Therefore $\xi$ is in the kernel of $H^{1}\left(k_{v}, E[m]\right) \rightarrow H^{1}\left(k_{v}, E\right)[m]$ which is equal to $\operatorname{Im} \delta_{v}$, hence $\operatorname{Im} \delta_{v}^{\perp} \subset \operatorname{Im} \delta_{v}$, consequently we have

$$
\operatorname{Im} \delta_{v}=\operatorname{Im} \delta_{v}^{\perp}
$$




\section{The cases for odd primes.}

In this section, we will embed the Selmer group $S^{(p)}(E / k)$ for an odd prime number $p$ in some Galois cohomology group under some assumptions, and estimate the order of $S^{(p)}(E / k)$ by making use of the genus formula.

Let assume that $E(k)$ contains the $p$-torsion subgroup $E[p]$. Moreover we assume that the following condition (A) holds.

(A) There are such generators $P_{1}, P_{2}$ of $p^{2}$-torsion subgroup $E\left[p^{2}\right]$ over $\mathbf{Z} / p^{2} \mathbf{Z}$ that the definition fields $K_{1}=k\left(P_{1}\right), K_{2}=k\left(P_{2}\right)$ of $P_{1}, P_{2}$ over $k$ are both cyclic extensions of degree $p$ over $k$, and if we put

$$
G_{i}=\operatorname{Gal}\left(K_{i} / k\right)=\left\langle\tau_{i}\right\rangle \quad(i=1,2),
$$

then

$$
P=P_{1}^{\tau_{1}}-P_{1}, \quad P^{\prime}=P_{2}^{\tau_{2}}-P_{2}
$$

generate $E[p]$ over $\mathbf{Z} / p \mathbf{Z}$.

We take the above generators $P, P^{\prime}$ of $E[p]$ in order to define the map $j$ in Section 2 and consider the following maps

$$
E(k) / p E(k) \stackrel{\delta}{\longrightarrow} H^{1}(k, E[p]) \stackrel{j}{\sim}\left(k^{\times} / k^{\times p}\right)^{2} .
$$

Then by the definition of $\delta$ and the condition (A), for any $\sigma \in \mathrm{Gal}(k / k)$

$$
\delta\left([p] P_{1}\right)(\sigma)=P_{1}^{\sigma}-P_{1} \in\langle P\rangle .
$$

Hence, the second component of the image $i \circ \delta\left([p] P_{1}\right)$ becomes always trivial:

$$
\left(i \circ \delta\left([p] P_{1}\right)\right)_{2}(\sigma)=e_{p}\left(P, P_{1}^{\sigma}-P_{1}\right)=1 \quad \text { for any } \sigma \in \operatorname{Gal}(\bar{k} / k) .
$$

Therefore the image $j \circ \delta\left([p] P_{1}\right)$ is always in the form

$$
j \circ \delta\left([p] P_{1}\right)=\left(a_{1}, 1\right) \in\left(k^{\times} / k^{\times p}\right)^{2}
$$

for some $a_{1} \in k^{\times} / k^{\times p}$. Similarly, the image of $[p] P_{2}$ is in the form

$$
j \circ \delta\left([p] P_{2}\right)=\left(1, a_{2}\right) \in\left(k^{\times} / k^{\times p}\right)^{2}
$$

for some $a_{2} \in k^{\times} / k^{\times p}$. For any $v \in M_{k}$, the images of $j_{v} \circ \delta_{v}$ are also given by

$$
\left\{\begin{array}{l}
j_{v} \circ \delta_{v}\left([p] P_{1}\right)=\left(a_{1}, 1\right) \in\left(k_{v}^{\times} / k_{v}^{\times p}\right)^{2}, \\
j_{v} \circ \delta_{v}\left([p] P_{2}\right)=\left(1, a_{2}\right) \in\left(k_{v}^{\times} / k_{v}^{\times p}\right)^{2} .
\end{array}\right.
$$

On the other hand, let $L / K$ be an arbitrary field extension. Then for an elliptic curve $E / K$, the following diagram is commutative: 


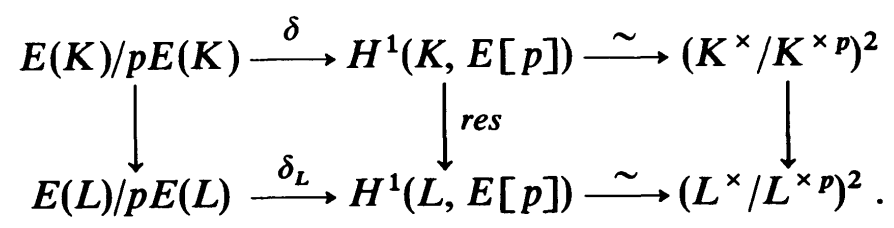

For any $P \in E(K), K\left([p]^{-1} P\right)$ is contained in $L$ if and only if $P$ is contained in $p E(L)$, and it is equivalent to that $\delta(P)$ is an element of the kernel of the map $\left(K^{\times} / K^{\times p}\right)^{2} \rightarrow\left(L^{\times} / L^{\times p}\right)^{2}$. Since the kernel is $\left(\left(K^{\times} \cap L^{\times p}\right) / K^{\times p}\right)^{2}$, if we put $\delta(P)=\left(\delta(P)_{1}\right.$, $\left.\delta(P)_{2}\right)$, it is equivalent to that $K\left(\sqrt[p]{\delta(P)_{1}}, \sqrt[p]{\delta(P)_{2}}\right)$ is contained in $L$. Therefore we have

$$
K\left([p]^{-1} P\right)=K\left(\sqrt[p]{\delta(P)_{1}}, \sqrt[p]{\delta(P)_{2}} \quad \text { for any } P \in E(K)\right. \text {. }
$$

Consequently, we have

$$
K_{1}=k\left(P_{1}\right)=k\left(\sqrt[p]{a_{1}}\right), \quad K_{2}=k\left(P_{2}\right)=k\left(\sqrt[p]{a_{2}}\right) .
$$

Moreover, it is clear that the subgroups of $\left(k_{v}^{\times} / k_{v}^{\times p}\right)^{2}$ generated by $\left(a_{1}, 1\right),\left(1, a_{2}\right)$, denoted by $\left\langle\left(a_{1}, 1\right)\right\rangle_{v},\left\langle\left(1, a_{2}\right)\right\rangle_{v}$ respectively, are contained in $\operatorname{Im} \delta_{v}$. Hence, taking the annihilators with respect to the bilinear map $\Phi:\left(k_{v}^{\times} / k_{v}^{\times p}\right)^{2} \times\left(k_{v}^{\times} / k_{v}^{\times p}\right)^{2} \rightarrow \mu_{p}$ and by (1), we have

$$
\operatorname{Im} \delta_{v}=\operatorname{Im} \delta_{v}^{\perp} \subset\left\langle\left(a_{1}, 1\right)\right\rangle_{v}^{\perp}, \quad \operatorname{Im} \delta_{v}=\operatorname{Im} \delta_{v}^{\perp} \subset\left\langle\left(1, a_{2}\right)\right\rangle_{v}^{\perp} .
$$

Let $(c, d) \in\left(k_{v}^{\times} / k_{v}^{\times p}\right)^{2}$ be an annihilator of $\left(a_{1}, 1\right)$. Then by the definition we see

$$
1=\Phi\left(\left(a_{1}, 1\right),(c, d)\right)=\left(a_{1}, d\right)_{v}(1, c)_{v}^{-1}=\left(a_{1}, d\right)_{v} .
$$

Hence $d$ must be an annihilator of $a_{1}$ with respect to the Hilbert norm residue symbol and $c$ is arbitrary. In $k_{v}^{\times} / k_{v}^{\times p}$ the Kummer group for $K_{1, \omega}=k_{v}\left(\sqrt[p]{a_{1}}\right)$ is the subgroup generated by $a_{1}$, denoted by $\left\langle a_{1}\right\rangle_{v}$, and its annihilator with respect to the Hilbert norm residue symbols is the norm of $K_{1, \omega}^{\times}$, namely $N K_{1, \omega}^{\times} k_{v}^{\times p} / k_{v}^{\times p}$. Therefore we have

$$
\left\langle\left(a_{1}, 1\right)\right\rangle_{v}^{\perp}=k_{v}^{\times} / k_{v}^{\times p} \times N K_{1, \omega}^{\times} k_{v}^{\times p} / k_{v}^{\times p} .
$$

Similarly

$$
\left\langle\left(1, a_{2}\right)\right\rangle_{v}^{\perp}=N K_{2, \omega^{\prime}}^{\times} k_{v}^{\times p} / k_{v}^{\times p} \times k_{v}^{\times} / k_{v}^{\times p},
$$

where $K_{2, \omega^{\prime}}=k_{v}\left(\sqrt[p]{a_{2}}\right)$. Hence we have an inclusion

$$
\begin{aligned}
\operatorname{Im} \delta_{v} & \subseteq\left\langle\left(a_{1}, 1\right)\right\rangle_{v}^{\perp} \cap\left\langle\left(1, a_{2}\right)\right\rangle_{v}^{\perp} \\
& =N K_{2, \omega}^{\times} k_{v}^{\times p} / k_{v}^{\times p} \times N K_{1, \omega}^{\times} k_{v}^{\times p} / k_{v}^{\times p} .
\end{aligned}
$$

Let $\mathcal{O}_{1, \omega}$ denote the ring of integers of $K_{1, \omega}$. Then if $K_{1, \omega} / k_{v}$ is unramified, we have

$$
N K_{1, \omega}^{\times} \equiv N \mathcal{O}_{1, \omega}^{\times} \quad\left(\bmod k_{v}^{\times p}\right) .
$$

Similarly, if $K_{2, \omega^{\prime}}^{\times} / k_{v}$ is unramified, then

$$
N K_{2, \omega^{\prime}}^{\times} \equiv N \mathcal{O}_{2, \omega^{\prime}}^{\times} \quad\left(\bmod k_{v}^{\times p}\right) .
$$


Let $R_{i}$ be the set of places of $k$ which are ramified in $K_{i}(i=1,2)$. Moreover we put $T_{i}=R_{i} \cap S(i=1,2)$, where $S$ means the same set as in Section 2 . Let $T_{i}^{\prime}$ be the set of places of $K_{i}$ lying above places of $T_{i}$. Then $T_{i}^{\prime}$-idèle group $J_{K_{i}, T_{i}^{\prime}}$ of $K_{i}$ is defined by

$$
J_{K_{i}, T_{i}^{\prime}}:=\prod_{\omega \in M_{K_{i}} \backslash T_{i}^{\prime}} \mathcal{O}_{i, \omega}^{\times} \times \prod_{\omega \in T_{i}^{\prime}} K_{i, \omega}^{\times},
$$

where for an infinite place $\omega, \mathcal{O}_{i, \omega}^{\times}$denotes $\mathbf{C}^{\times}$. Note that by the assumption, $k$ contains $\mu_{p}$, hence $k$ and $K_{i}$ are totally imaginary.

For each places $v$ of $k$, we choose and fix a place $\omega$ of $K_{i}$ lying above $v$ once for all. Then by the semi-local theory, there exists an isomorphism

$$
\hat{H}^{0}\left(K_{i} / k, J_{K_{i}, T_{i}^{\prime}} \cong \underset{v \notin T_{i}}{\bigoplus} \hat{H}^{0}\left(K_{i, \omega} / k_{v}, \mathcal{O}_{i, \omega}^{\times}\right) \oplus \underset{v \in T_{i}}{\bigoplus} \hat{H}^{0}\left(K_{i, \omega} / k_{v}, K_{i, \omega}^{\times}\right),\right.
$$

where $\hat{H}$ means the Tate cohomology.

Let $f$ be the composition of the following maps.

$$
\begin{aligned}
\prod_{i=1,2} H^{1}\left(k_{T_{i}} / k, \mu_{p}\right) \stackrel{\prod\left(\prod_{v \in T_{i} r e s_{v}}\right)}{\longrightarrow} & \prod_{i=1,2}\left(\sum_{v \in T_{i}} H^{1}\left(k_{T_{i}, v} / k_{v}, \mu_{p}\right)\right) \\
\longrightarrow & \prod_{i=1,2}\left(\prod_{v \in T_{i}} \hat{H}^{0}\left(K_{i, \omega} / k_{v}, K_{i, \omega}^{\times}\right)\right) \\
& \hookrightarrow \prod_{i=1,2}\left(\hat{H}^{0}\left(K_{i} / k, J_{K_{i}, T_{i}^{\prime}}\right)\right)
\end{aligned}
$$

Then we have

LEMMA 2. There is an inclusion

$$
S^{(p)}(E / k) \subset \operatorname{Ker}\left\{\prod_{i=1,2} H^{1}\left(k_{T_{i}} / k, \mu_{p}\right) \stackrel{f}{\longrightarrow} \prod_{i=1,2} \hat{H}^{0}\left(K_{i} / k, J_{K_{i}, T_{i}^{\prime}}\right)\right\} .
$$

Proof. Looking at the following diagram

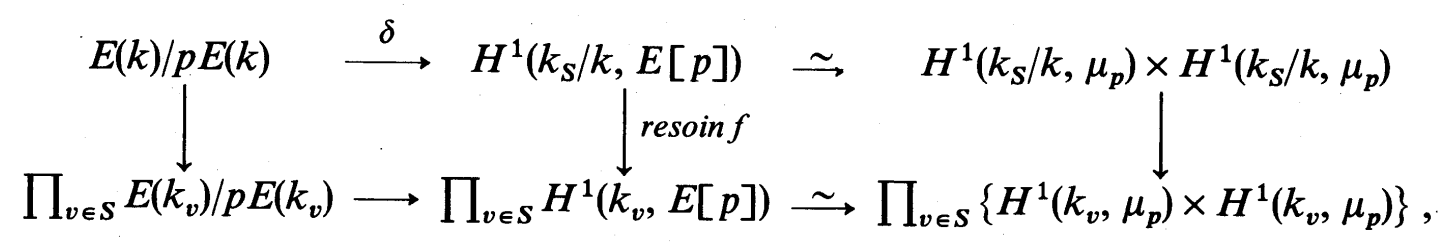

the Selmer group $S^{(p)}(E / k)$ can be expressed as

$$
S^{(p)}(E / k)=\left\{\left(\xi_{1}, \xi_{2}\right) \in H^{1}\left(k_{S} / k, \mu_{p}\right)^{2} \mid \operatorname{res}_{v}\left(\xi_{1}, \xi_{2}\right) \in \operatorname{Im}\left(i_{v} \circ \delta_{v}\right) \text { for any } v \in S\right\} .
$$

Since the map $i_{v}$ is an isomorphism, we identify $\operatorname{Im} \delta_{v}$ with $\operatorname{Im}\left(i_{v} \circ \delta_{v}\right)$. Then, by (2), (3) and (4), we have for each $\left(\xi_{1}, \xi_{2}\right) \in S^{(p)}(E / k)$

$$
\begin{array}{ll}
\operatorname{res}_{v} \xi_{1} \in N \mathcal{O}_{2, \omega}^{\times} k_{v}^{\times p} / k_{v}^{\times p} & \left(v \in S \backslash T_{2}\right), \\
\operatorname{res}_{v} \xi_{2} \in N \mathcal{O}_{1, \omega}^{\times} k_{v}^{\times p} / k_{v}^{\times p} & \left(v \in S \backslash T_{1}\right) .
\end{array}
$$


On the other hand, by Kummer theory, there is an isomorphism

$$
H^{1}\left(k_{S} / k, \mu_{p}\right) \cong\left\{x \in k^{\times} / k^{\times p} \mid \operatorname{ord}_{v}(x) \equiv 0(\bmod p) \text { for any } v \notin S\right\}
$$

Hence we have

$$
\begin{cases}\operatorname{ord}_{v}\left(\operatorname{res}_{v} \xi_{1}\right) \equiv 0(\bmod p) & \text { for any } v \in T_{2}, \\ \operatorname{ord}_{v}\left(\operatorname{res}_{v} \xi_{2}\right) \equiv 0(\bmod p) & \text { for any } v \notin T_{1}\end{cases}
$$

Therefore we have an inclusion

$$
\begin{aligned}
S^{(p)}(E / k) \subseteq & H^{1}\left(k_{T_{2}} / k, \mu_{p}\right) \times H^{1}\left(k_{T_{1}} / k, \mu_{p}\right) \\
\cong & \left\{x \in k^{\times} / k^{\times p} \mid \operatorname{ord}_{v}(x) \equiv 0(\bmod p) \text { for any } v \notin T_{2}\right\} \\
& \times\left\{x \in k^{\times} / k^{\times p} \mid \operatorname{ord}_{v}(x) \equiv 0(\bmod p) \text { for any } v \notin T_{1}\right\} .
\end{aligned}
$$

Moreover, for $v \in T_{2}$

$$
\operatorname{res}_{v} \xi_{1} \in N K_{2, \omega^{\prime}}^{\times} k_{v}^{\times p} / k_{v}^{\times p}=\operatorname{Ker}\left\{k_{v}^{\times} / k_{v}^{\times p} \rightarrow \hat{H}^{\mathrm{O}}\left(K_{2, \omega^{\prime}} / k_{v}, K_{2, \omega^{\prime}}^{\times}\right)\right\} .
$$

Similarly, for $v \in T_{1}$

$$
\operatorname{res}_{v} \xi_{2} \in N K_{1, \omega}^{\times}, k_{v}^{\times p} / k_{v}^{\times p}=\operatorname{Ker}\left\{k_{v}^{\times} / k_{v}^{\times p} \rightarrow \hat{H}^{0}\left(K_{1, \omega} / k_{v}, K_{1, \omega}^{\times}\right)\right\} .
$$

This gives the desired inclusion.

In general, let $K / k$ be any finite Galois extension and $G=\mathrm{Gal}(K / k)$ be the Galois group. Then for a finite subset $T$ of $M_{k}, T$-unit group $U_{k, T}$ of $k$ is defined by

$$
\begin{aligned}
& U_{k, T}=\left\{u \in k^{\times} \mid v(u)>0 \text { for all real } v \in M_{k}^{\infty} \backslash T\right. \text { and } \\
& \left.\qquad \operatorname{ord}_{v}(u)=0 \text { for all } v \in M_{k}^{0} \backslash T\right\},
\end{aligned}
$$

where $M_{k}^{\infty}\left(\right.$ resp. $M_{k}^{0}$ ) is the set of all infinite (resp. finite) places of $k$. Let $T^{\prime}$ be the set of places of $K$ lying above the places of $T$. Then the $T^{\prime}$-unit group $U_{K, T^{\prime}}$ is also defined in a similar way. Let $J_{K, T^{\prime}}$ be the $T^{\prime}$-idèle group of $K$, and $C_{K, T^{\prime}}$ be a group defined by the following exact sequence

$$
0 \longrightarrow U_{K, T^{\prime}} \stackrel{\alpha}{\longrightarrow} J_{K, T^{\prime}} \stackrel{\beta}{\longrightarrow} C_{K, T^{\prime}} \longrightarrow 0,
$$

where $\alpha$ denotes the diagonal embedding.

Taking the Tate cohomology we obtain a long exact sequence

$$
\begin{aligned}
\cdots & \longrightarrow \hat{H}^{0}\left(G, U_{K, T^{\prime}}\right) \stackrel{\alpha_{0}}{\longrightarrow} \hat{H}^{0}\left(G, J_{K, T^{\prime}}\right) \stackrel{\beta_{0}}{\longrightarrow} \hat{H}^{0}\left(G, C_{K, T^{\prime}}\right) \\
& \longrightarrow H^{1}\left(G, U_{K, T^{\prime}} \stackrel{\alpha_{1}}{\longrightarrow} H^{1}\left(G, J_{K, T^{\prime}}\right) \stackrel{\beta_{1}}{\longrightarrow} H^{1}\left(G, C_{K, T^{\prime}}\right)\right. \\
& \longrightarrow H^{2}\left(G, U_{K, T^{\prime}}\right) \stackrel{\alpha_{2}}{\longrightarrow} H^{2}\left(G, J_{K, T^{\prime}}\right) \stackrel{\beta_{2}}{\longrightarrow} H^{2}\left(G, C_{K, T^{\prime}}\right)
\end{aligned}
$$


Let $C l_{k, T}$ (resp. $C l_{K, T^{\prime}}$ ) be the group $J_{k} / k^{\times} J_{k, T}$ (resp. $J_{K} / K^{\times} J_{K, T^{\prime}}$ ). Suppose that $T$ contains all the places which ramify in $K / k$, and that $K / k$ is a cyclic extension, then Aoki [1] shows the following genus formula:

Suppose that $K / k$ is a cyclic extension. Then it holds that

$$
\left|\operatorname{Ker} \alpha_{2}\right|=\frac{[K: k]\left|\hat{H}^{0}\left(G, U_{K, T^{\prime}}\right)\right|}{e f_{T}} \frac{\left|\left(C l_{K, T^{\prime}}\right)^{G}\right|}{\left|C l_{k, T}\right|},
$$

where $e=\prod_{v \in M_{k}} e_{v}$ is the product of the relative ramification indeces in $K / k$ and $f_{T}=\prod_{v \in T} f_{v}$ is the product of the relative degree of $v \in T$ in $K / k$ [1].

In our case, the $T_{i}$-unit group of $k$ is given by

$$
U_{k, T_{i}}=\left\{u \in k^{\times} \mid \operatorname{ord}_{v}(u)=0 \text { for any } v \in M_{k}^{0} \backslash T_{i}\right\} \quad(i=1,2) .
$$

The $T_{i}^{\prime}$-unit group of $K_{i}$ is also given in a similar form.

We define a map

$$
\lambda_{i}: U_{k, T_{i}} k^{\times p} / k^{\times p} \longrightarrow \hat{H}^{0}\left(K_{i} / k, J_{K_{i}, T_{i}^{\prime}}\right)
$$

by the composition of the natural surjection

$$
U_{k, T_{i}} k^{\times p} / k^{\times p}\left(\cong U_{k, T_{i}} / U_{k, T_{i}} \cap k^{\times p}\right) \longrightarrow U_{k, T_{i}} / N U_{K_{i}, T_{i}^{\prime}}\left(\cong \hat{H}^{0}\left(K_{i} / k, U_{K_{i}, T_{i}^{\prime}}\right)\right),
$$

where $N$ is the norm map from $K_{i}^{\times}$to $k^{\times}$, and

$$
\alpha_{0}^{(i)}: \hat{H}^{\mathrm{o}}\left(K_{i} / k, U_{K_{i}, T_{i}^{\prime}}\right) \longrightarrow \hat{H}^{\mathrm{o}}\left(K_{i} / k, J_{K_{i}, T_{i}^{\prime}}\right),
$$

where $\alpha_{r}^{(i)}$ 's are the maps obtained from taking cohomology groups for the exact sequence

$$
0 \longrightarrow U_{K_{i}, T_{i}^{\prime}} \stackrel{\alpha^{(i)}}{\longrightarrow} J_{K_{i}, T_{i}^{\prime}} \stackrel{\beta^{(i)}}{\longrightarrow} C_{K_{i}, T_{i}^{\prime}} \longrightarrow 0
$$

as in (5), (6). Note that, since $K_{i} / k$ is a cyclic extension by the condition (A), $\hat{H}^{0}=H^{2}$ and $\alpha_{0}^{(i)}=\alpha_{2}^{(i)}$. Using the genus formula (7) in order to estimate the orders of the kernels of $\lambda_{i}$ 's, we obtain the following upper bound of the order of the Selmer group.

THEOREM 1. Assume that $E(k)$ contains $E[p]$ and the condition (A) holds. Then

$$
\left|S^{(p)}(E / k)\right| \leq p^{2+d-r} \frac{\left|\left(C l_{K_{1}, T_{1}^{\prime}}\right)^{G_{1}}\right|}{\left|\left(C l_{k, T_{1}}\right)^{p}\right|} \frac{\left|\left(C l_{K_{2}, T_{2}^{\prime}}\right)^{G_{2}}\right|}{\left|\left(C l_{k, T_{2}}\right)^{p}\right|},
$$

where $d=[k: \mathbf{Q}]$ and $r=\left|R_{1} \backslash T_{1}\right|+\left|R_{2} \backslash T_{2}\right|$.

Proof. By the definition, the order of the kernel of $\lambda_{i}$ can be written as

$$
\left|\operatorname{Ker} \lambda_{i}\right|=\frac{\left|U_{k, T_{i}} / U_{k, T_{i}}{ }^{p}\right|}{\left|\hat{H}^{0}\left(K_{i} / k, U_{K_{i}, T_{i}^{\prime}}\right)\right|}\left|\operatorname{Ker} \alpha_{2}^{(i)}\right| \quad(i=1,2) \text {. }
$$


By the Dirichlet's unit theorem, we have $\left|U_{k, T_{i}} / U_{k, T_{i}}{ }^{p}\right|=p^{d / 2+\left|T_{i}\right|}$ where $d=[k: \mathbf{Q}]$.

Let $C l_{k, T_{i}}$, be the group $J_{k} / k^{\times} J_{k, T_{i}}$ as above. Then, there exists an exact sequence

$$
0 \longrightarrow U_{k, T_{i}} k^{\times p} / k^{\times p} \longrightarrow H^{1}\left(k_{T_{i}} / k, \mu_{p}\right) \longrightarrow\left(C l_{k, T_{i}}\right)_{p} \longrightarrow 0
$$

where ( $)_{p}$ denotes the $p$-torsion subgroup. Moreover the next diagram is commutative:

$$
\begin{aligned}
& 0 \longrightarrow \prod_{i=1,2} U_{K_{i}, T_{i}^{\prime}} k^{\times p} / k^{\times p} \longrightarrow \prod_{i=1,2} H^{1}\left(k_{T_{i}} / k, \mu_{p}\right) \longrightarrow \prod_{i=1,2}\left(C l_{k, T_{i}}\right)_{p} \longrightarrow 0 \\
& \downarrow \lambda:=\prod \lambda_{i} \quad \downarrow f \\
& 0 \longrightarrow \prod_{i=1,2} \hat{H}^{0}\left(K_{i} / k, J_{K_{i}, T_{i}^{\prime}}\right) \stackrel{i d .}{\longrightarrow} \prod_{i=1,2} \hat{H}^{0}\left(K_{i} / k, J_{K_{i}, T_{i}^{\prime}}\right) \longrightarrow \quad 0 \quad 0 \quad \longrightarrow 0
\end{aligned}
$$

where $f$ is defined in Lemma 2. Then, by the Snake Lemma we see

$$
|\operatorname{Ker} f|=\frac{\left|\operatorname{Coker} f\left\|\left(C l_{k, T_{1}}\right)_{p}\right\|\left(C l_{k, T_{2}}\right)_{p}\right||\operatorname{Ker} \lambda|}{|\operatorname{Coker} \lambda|} .
$$

Since $\mid$ Coker $\lambda|\geq|$ Coker $f \mid$, we obtain

$$
\left|S^{(p)}(E / k)\right| \leq|\operatorname{Ker} f| \leq\left|\left(C l_{k, T_{1}}\right)_{p} \|\left(C l_{k, T_{2}}\right)_{p}\right||\operatorname{Ker} \lambda| \text {. }
$$

On the other hand, by the genus formula, we have

$$
\left|\operatorname{Ker} \alpha_{2}^{(i)}\right|=\frac{[K: k]\left|\hat{H}^{0}\left(K_{i} / k, U_{K_{i}, T_{i}^{\prime}}\right)\right|}{e_{i}} \frac{\left|\left(C l_{K_{i}, T_{i}^{\prime}}\right)^{G_{i}}\right|}{\left|C l_{k, T_{i}}\right|} \quad(i=1,2),
$$

where $e_{i}=\prod_{v \in M_{k}} e_{v}$ is the product of relative ramification indices in $K_{i} / k$. Note that, since $K_{i} / k$ is a cyclic extension of degree $p$ by condition (A), the residue class degrees are equal to 1 for any $v \in T_{i}$. Hence we have

$$
\begin{aligned}
|\operatorname{Ker} \lambda| & =\left|\operatorname{Ker} \lambda_{1}\right|\left|\operatorname{Ker} \lambda_{2}\right| \\
& =\frac{p^{2+d+\left|T_{1}\right|+\left|T_{2}\right|}}{e_{1} e_{2}} \frac{\left|\left(C l_{K_{1}, T_{1}^{\prime}}\right)^{G_{1}}\right|}{\left|C l_{k, T_{1}}\right|} \frac{\left|\left(C l_{K_{2}, T_{2}^{\prime}}\right)^{G_{2}}\right|}{\left|C l_{k, T_{2}}\right|} .
\end{aligned}
$$

Since $\left|C l_{k, T_{i}}\right| /\left|\left(C l_{k, T_{i}}\right)_{p}\right|=\left|\left(C l_{k, T_{i}}\right)^{p}\right|$, and $e_{i}=p^{\left|R_{i}\right|}$, we obtain the desired estimate

$$
\left|S^{(p)}(E / k)\right| \leq p^{2+d-r} \frac{\left|\left(C l_{K_{1}, T_{1}^{\prime}}\right)^{G_{1}}\right|}{\left|\left(C l_{k, T_{1}}\right)^{p}\right|} \frac{\left|\left(C l_{K_{2}, T_{2}^{\prime}}\right)^{G_{2}}\right|}{\left|\left(C l_{k, T_{2}}\right)^{p}\right|}
$$

Let $\operatorname{dim}_{p}$ denote the dimension over $\mathbf{Z} / p \mathbf{Z}$. By the assumption, we have $\operatorname{rank} E(k)=\operatorname{dim}_{p} E(k) / p E(k)-2$, and there is an injection $\delta: E(k) / p E(k) \rightarrow S^{(p)}(E / k)$. Hence we have the following

COROLlary. We have an inequality

$$
\operatorname{rank} E(k) \leq d-r+\operatorname{ord}_{p} \frac{\left|\left(C l_{\boldsymbol{K}_{1}, \boldsymbol{T}_{1}^{\prime}}\right)^{\boldsymbol{G}_{1}}\right|}{\left|\left(C l_{k, T_{1}}\right)^{p}\right|}+\operatorname{ord}_{p} \frac{\left|\left(C l_{\boldsymbol{K}_{2}, \boldsymbol{T}_{2}^{\prime}}\right)^{\mathbf{G}_{2}}\right|}{\left|\left(C l_{k, T_{2}}\right)^{p}\right|} .
$$




\section{The case for $p=2$.}

In the case of $p=2$, the situation on the infinite places of $k$ is slightly different from the one for odd primes. For an odd prime $p$, the condition $E[p] \subset E(k)$ implies that $k$ is a totally imaginary number filed. On the other hand, we deduce nothing about the infinite places $k$ from the condition $E[2] \subset E(k)$. However, if we make some assumption on the infinite places in order to simplify the situation (for example, $k$ is totally imaginary), then we can estimate the 2-Selmer group for $E / k$ in a similar way for odd primes. In the case that $k$ is a totally real number field, we also obtain a similar estimate as follows.

Let $k$ be a totally real number field, $E$ be an elliptic curve defined over $k$. Let assume that $E[2] \subset E(k)$ and the following condition (B) holds.

(B) There are such generators $P_{1}, P_{2}$ of 4-torsion subgroup $E$ [4] over $\mathrm{Z} / 4 \mathrm{Z}$ that the definition fields $K_{1}=k\left(P_{1}\right), K_{2}=k\left(P_{2}\right)$ of $P_{1}, P_{2}$ over $k$ are both cyclic extensions of degree 2 over $k$, and if we put

$$
G_{i}=\operatorname{Gal}\left(K_{i} / k\right)=\left\langle\tau_{i}\right\rangle \quad(i=1,2),
$$

then

$$
P=P_{1}^{\tau_{1}}-P_{1}, \quad P^{\prime}=P_{2}^{\tau_{2}}-P_{2}
$$

generate $E[2]$ over $\mathbf{Z} / 2 \mathbf{Z}$.

This is the same condition as (A) for $p=2$ in Section 3.

Let $R_{i}$ be the set of places of $k$ which ramify in $K_{i}(i=1,2)$, and put

$$
T_{i}^{(2)}:=\left(R_{i} \cap S\right) \cup\{\text { the places lying above } 2\} \cup M_{k}^{\infty} \text {. }
$$

THEOREM 2. Under the above conditions we have

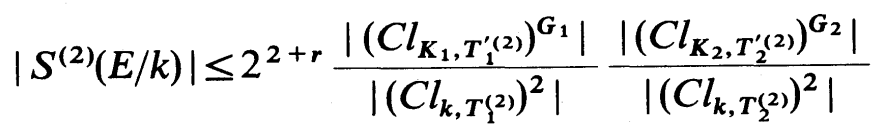

where $r:=\left|T_{1}^{(2)}\right|+\left|T_{2}^{(2)}\right|-\left|R_{1}\right|-\left|R_{2}\right|$.

Proof. Each element of $S \backslash T_{i}^{(2)}(i=1,2)$ is a finite place. Hence the argument is completely similar to the one for odd primes. Note that since $T_{i}^{(2)}$ contains all infinite places of $k$, for the $T_{i}^{(2)}$-unit group $U_{k, T_{i}^{(2)}}$, the order of the group $U_{k, T_{i}^{(2)}} / U_{k, T_{i}^{(2)}}^{2}$ is equal to $2^{\left|T_{i}^{(2)}\right|}$ by Dirichlet's theorem.

\section{Elliptic curves without complex multiplication.}

In this section, we show that if an elliptic curve has no complex multiplication, then choosing some prime number $p$ and replacing $k$ by its finite extension if necessary, 
we can make the condition (A) in Section 3 to be valid.

Let $E / k$ be an elliptic curve defined over a number field of finite degree. For any integer $m \geq 2, E[m]$ is a free $(\mathbf{Z} / m \mathbf{Z})$-module of rank 2, and $G(m)=\mathrm{Gal}(k(m) / k)$ acts on $E[m]$ where $k(m)=k(E[m])$. Hence by taking some generators of $E[m]$ over $\mathbf{Z} / m \mathbf{Z}$, there exists a homomorphism $G(m) \rightarrow G L_{2}(\mathrm{Z} / m \mathrm{Z})$. Let us assume that $E$ has no complex multiplication. Then by Serre [6], [8], we know that for all but finitely many prime numbers $p$, the above homomorphisms are isomorphisms:

$$
G\left(p^{n}\right) \stackrel{\sim}{\sim} G L_{2}\left(\mathbf{Z} / p^{n} \mathbf{Z}\right) \quad \text { for any } n \geq 1 \text {. }
$$

From an elementary fact, the orders of $G L_{2}(\mathbf{Z} / p \mathbf{Z})$ and $G L_{\mathbf{2}}\left(\mathbf{Z} / p^{2} \mathbf{Z}\right)$ can be given by

$$
\left|G L_{2}(\mathbf{Z} / p \mathbf{Z})\right|=p\left(p^{2}-1\right)(p-1), \quad\left|G L_{2}\left(\mathbf{Z} / p^{2} \mathbf{Z}\right)\right|=p^{5}\left(p^{2}-1\right)(p-1) .
$$

Hence, for any prime $p$ which satisfies (8), $\left[k\left(p^{2}\right): k(p)\right]=p^{4}$. Let $P_{1}, P_{2} \in E\left[p^{2}\right]$ be any generators of $E\left[p^{2}\right]$ over $\mathbf{Z} / p^{2} \mathbf{Z}$. Then it is easily seen that for $\sigma \in G\left(p^{2}\right), \sigma$ fixes $E[p]$ and $\mu_{p^{2}}$ if and only if $\left([p] P_{1}\right)^{\sigma}=[p] P_{1},\left([p] P_{2}\right)^{\sigma}=[p] P_{2}$ and $\sigma \in S L_{2}\left(Z / p^{2} Z\right)$, because $[p] P_{1}$ and $[p] P_{2}$ generate $E[p]$ over $\mathbf{Z} / p \mathbf{Z}$. We rewrite this condition in terms of matrices. First, since $\sigma$ fixes $[p] P_{1}$ and $[p] P_{2}$, putting $\sigma=\left(\begin{array}{ll}a & b \\ c & d\end{array}\right), a, b, c, d \in \mathbf{Z} / p^{2} \mathbf{Z}$,

$$
\left(\begin{array}{l}
p \\
0
\end{array}\right)=\left(\begin{array}{ll}
a & b \\
c & d
\end{array}\right)\left(\begin{array}{l}
p \\
0
\end{array}\right)=\left(\begin{array}{l}
a p \\
c p
\end{array}\right), \quad\left(\begin{array}{l}
0 \\
p
\end{array}\right)=\left(\begin{array}{ll}
a & b \\
c & d
\end{array}\right)\left(\begin{array}{l}
0 \\
p
\end{array}\right)=\left(\begin{array}{l}
b p \\
d p
\end{array}\right) .
$$

Hence we have $a \equiv d \equiv 1(\bmod p)$ and $b \equiv c \equiv 0(\bmod p)$. Then if we put $\sigma=\left(\begin{array}{cc}1+p \alpha & p \beta \\ p \gamma & 1+p \eta\end{array}\right)$ for some $\alpha, \beta, \gamma, \eta \in \mathbf{Z} / p^{2} \mathbf{Z}$, since $\sigma$ is in $S L_{2}\left(\mathbf{Z} / p^{2} \mathbf{Z}\right), \eta$ must be equal to $-\alpha$. Therefore $\sigma \in H:=\operatorname{Gal}\left(k\left(p^{2}\right) /(k(p))\left(\mu_{p^{2}}\right)\right)$ if and only if it is in the form

$$
\sigma=\left(\begin{array}{cc}
1+p \alpha & p \beta \\
p \gamma & 1-p \alpha
\end{array}\right) \quad \text { for some } \alpha, \beta, \gamma \in \mathbf{Z} / p^{2} \mathbf{Z} .
$$

The number of such $\sigma^{\prime}$ s is $p^{3}$, namely $|H|=p^{3}$, hence $\left[(k(p))\left(\mu_{p^{2}}\right): k(p)\right]=p$. Moreover, the subgroup of $H$ consisting of the elements which fix $P_{1}$ is a group generated by $\sigma_{1}=\left(\begin{array}{ll}1 & p \\ 0 & 1\end{array}\right)$. Similarly the subgroup which fixes $P_{2}$ is generated by $\sigma_{2}=\left(\begin{array}{ll}1 & 0 \\ p & 1\end{array}\right)$ and their orders are both $p$. Hence if we put

$$
K=(k(p))\left(\mu_{p^{2}}, P_{1}\right) \cap(k(p))\left(\mu_{p^{2}}, P_{2}\right),
$$

then $K\left(P_{1}\right)$ and $K\left(P_{2}\right)$ are both cyclic extensions of $K$ whose Galois Groups are generated by $\sigma_{2}$ and $\sigma_{1}$ respectively:

$$
\begin{aligned}
& G_{1}=\operatorname{Gal}\left(K\left(P_{1}\right) / K\right)=\left\langle\sigma_{2}\right\rangle=\left\langle\left.\sigma_{2}\right|_{K\left(P_{1}\right)}\right\rangle, \\
& G_{2}=\operatorname{Gal}\left(K\left(P_{2}\right) / K\right)=\left\langle\sigma_{1}\right\rangle=\left\langle\left.\sigma_{1}\right|_{K\left(P_{2}\right)}\right\rangle .
\end{aligned}
$$


Moreover,

$$
\begin{aligned}
& P_{1}^{\sigma_{2}}=\left(\begin{array}{ll}
1 & 0 \\
p & 1
\end{array}\right)\left(\begin{array}{l}
1 \\
0
\end{array}\right)=\left(\begin{array}{l}
1 \\
p
\end{array}\right)=P_{1}+[p] P_{2}, \\
& P_{2}^{\sigma_{1}}=\left(\begin{array}{ll}
1 & p \\
0 & 1
\end{array}\right)\left(\begin{array}{l}
0 \\
1
\end{array}\right)=\left(\begin{array}{l}
p \\
1
\end{array}\right)=[p] P_{1}+P_{2} .
\end{aligned}
$$

Hence $P_{1}^{\sigma_{2}}-P_{1}=[p] P_{2}$ and $P_{2}^{\sigma_{1}}-P_{2}=[p] P_{1}$ generate $E[p]$ over $\mathbf{Z} / p \mathbf{Z}$. Therefore we obtain the following.

Proposition. Let $E / k$ be an elliptic curve without complex multiplication, and $p$ be a prime number which satisfies the condition (8). Then there exists a finite extension field $K$ of $k$ such that the condition (A) holds for $E / K$ and $p$.

\section{References}

[ 1 ] N. AoKı, Selmer groups and ideal class groups, Comment. Math. Univ. St. Paul. 42 (1993), 209-229.

[2] A. Brumer and K. Kramer, The rank of elliptic curves, Duke Math. J. 44 (1977), 715-743.

[3] M. KAWACHI and S. NAKano, The 2-class groups of cubic fields and 2-descents on elliptic curves, Tôhoku Math. J. 44 (1992), 557-565.

[4] W. G. MCCAllum, On the Shafarevich-Tate group of the jacobian of a quotient of the Fermat curve, Invent. Math. 93 (1988), 637-666.

[ 5] J. S. Milne, Arithmetic Duality Theorems, Academic Press (1986).

[6] J.-P. SERre, Abelian l-Adic Representation and Elliptic Curves, Benjamin (1968).

[7] J.-P. SERrE, Local Fields, Springer (1979).

[ 8 ] J.-P. SERRE, Propriétés galoisiennes des points d'order fini des courbes elliptiques, Invent. Math. 15 (1972), 259-331.

[9] J. H. Silverman, The Arithmetic of Elliptic Curves, Springer (1986).

[10] J. TATE, Duality theorems in Galois cohomology over number fields, Proc. Internat. Cong. Math. Stockholm (1962), 288-295.

[11] L. C. Washington, Class numbers of the simplest cubic fields, Math. Computation 48 (1987), 371-384.

[12] L. C. Washington, Number fields and elliptic curves, Number Theory and Applications (R. A. Mollin ed.), Kluwer Academic Publishers (1989), 245-278.

Present Address:

Graduate School of Mathematics, Kyushu University 33,

Hakozaki, Fukuoka, 812 Japan. 